\title{
MARCO ANALÍTICO DEL SISTEMA DE AUTOBUSES DE TRÁNSITO RÁPIDO - BRT ${ }^{1}$
}

\section{ANALYTICAL FRAMEWORK FOR THE RAPID TRANSIT BUS SYSTEM - BRT}

\section{Madisson Yojan Carmona Rojas ${ }^{2}$}

\author{
"Voy a la ciudad \\ voy a trabajar \\ ahí está el placer \\ lo voy a buscar \\ (...) Cómo me llaman \\ eso no importa" \\ FRUKO Y SUS TESOS
}

\section{Resumen}

En este artículo se analizan los elementos fundamentales del aparato discursivo del sistema de transporte BRT (Autobuses de Tránsito Rápido). La aparición y expansión del BRT se enmarca aquí, en la crisis económica y urbana de la década del ochenta en América Latina. El análisis se desarrolla con base en la lectura de documentos producidos por las instituciones encargadas de promover la implementación del sistema de carriles confinados en las principales ciudades del continente. A lo largo del texto se hará mención a la implementación de este modelo del BRT que opera en la ciudad de México.

Palabras clave: Autobuses de Tránsito Rápido (BRT); Crisis Urbana; Metrobús; Ciudad De México.

\section{Abstract}

This article analyzes the fundamental elements of the discursive apparatus of the BRT (Bus Rapid Transit) transport system. The emergence and expansion of the BRT is part of the economic and urban crisis of the eighties in Latin America. The analysis is based on the reading of documents produced by the institutions in charge of promoting the implementation of the system of confined lanes in the main cities of the continent. Throughout the text will mention the implementation of this BRT model that operates in Mexico City.

Keywords: Bus Rapid Transit (BRT); Urban Crisis; Metrobús; Mexico City.

\footnotetext{
${ }^{1}$ Este artículo hace parte del desarrollo de la tesis de Maestría en Ciencias Sociales y Humanidades titulada: Orden urbano y Metrobús en la ciudad de México. El caso de la línea 1. Para la realización de este trabajo se contó con el apoyo del programa de becas de posgrado de la Universidad Autónoma Metropolitana de México (UAM) y el Consejo Nacional de Ciencia y Tecnología de México (Conacyt).

${ }^{2}$ Maestro en Ciencias Sociales y Humanidades por la Universidad Autónoma Metropolitana-Cuajimalpa de México. Profesor de la Licenciatura en Ciencias Sociales de la Universidad Pedagógica Nacional (UPN) y de la Universidad de Cundinamarca (UDEC) en Colombia. Coordinador de la Línea de Investigación en Geografía de la UPN. E-mail: mycarmonar@gmail.com
} 


\section{INTRODUCCIÓN}

Los sistemas de transporte del tipo Autobuses de Tránsito Rápido (BRT) en América Latina y particularmente en la ciudad de México, son consecuencia $-y$ a su vez manifestación- del "despliegue de un conjunto de tendencias y fenómenos" (De Mattos, 2001, p. 5) de gestión urbana que contribuyen a la definición de las políticas de desarrollo y planeación. Por lo anterior, se puede decir que se configura una geografía urbana que se manifestará en la transformación del orden urbano ${ }^{3}$.

En este sentido, para explicar la aparición del Metrobús y el papel de éste en la transformación del orden urbano en la ciudad de México, es necesario identificar los elementos nodales que integran el aparato discursivo de las actuales propuestas de movilidad urbana, en las que el BRT es uno de los pilares. Así pues, el presente artículo se encargará de explicar los elementos discursivos que a continuación se enuncian:

1. La demanda de sistemas de transporte sostenibles

2. Los modelos gestión mixtos (private-public partnership)

3. El impacto del fenómeno de la globalización en la gestión de las ciudades

4. La necesidad de un transporte público masivo más humano

Ahora bien, para comprender a cabalidad estos elementos discursivos, es importante ubicarlos en el marco de la crisis urbana de las décadas del ochenta y noventa, la cual, de acuerdo con autores como De Mattos, 2001; Graizbord, 2011; Balbo, 2003, es el escenario de donde emergen las tendencias y fenómenos urbanos que han contribuido en la actualidad a la transformación de las ciudades latinoamericanas, no solo en cuanto a su estructura espacial, sino también en relación con nuevos mecanismos de gobernanza y gestión.

\section{El escenario de la crisis urbana}

La segunda mitad del siglo pasado estuvo marcada para gran parte de los países de América Latina, por la puesta en marcha de modelos de industrialización de gran escala conocidos como de sustitución de importaciones. En este proceso, según lo explica Arturo Escobar en La Invención del tercer mundo (1998), el estado no solo actuó como "arquitecto" (p. 56) del desarrollo

\footnotetext{
3 Emilio Duhau y Ángela Giglia (2004), entienden este concepto: "un conjunto de normas y reglas tanto formales (pertenecientes a algunas jerarquía del orden jurídico) como convencionales a las que recurren los habitantes de la ciudad explícita o tácitamente en el desarrollo de práctica relacionadas con los usos y formas de apropiación de los espacios y bienes públicos de uso colectivo" (p. 258).
} 
económico sino además como cimiento de las ideas de progreso económico tan en boga en ese momento, y que emanaban, como el mismo autor lo refiere, tanto de la iniciativa nacional como de organismos multilaterales comprometidos con la lucha contra la pobreza y el atraso económico de los sectores de ingresos más bajos.

El papel de las ciudades en esta fase de industrialización fue fundamental, toda vez que es en ellas donde se construyeron los grandes complejos industriales que habrían de servir como soporte del desarrollo económico (Davis, 1999; Cuervo y González, 1998; Singer, 1975). Como consecuencia de ello, arriban a las urbes, alentadas por la creciente oferta de trabajo, grandes masas de habitantes de las áreas rurales que se asientan principalmente en sectores periféricos o en municipios cercanos; de esta forma, se empezará a configurar el panorama metropolitano que actualmente se puede apreciar.

En este punto se debe resaltar que este proceso de expansión de las ciudades si bien se encuentra condicionado por el desarrollo industrial, también obedeció, en casos como el de Colombia, a fenómenos de violencia política en el campo que presionaron a sus pobladores a buscar seguridad en las zonas urbanas (Viviescas, 1989; Alfonso, 2012). Además, se pueden apreciar, en el mismo periodo, procesos de movilidad interurbana en los cuales las ciudades con mayor producción industrial se constituyeron en fuertes polos de atracción de nuevos habitantes, como sucedió en el caso brasileño con San Pablo y Río de Janeiro, que pasaron de ser "regiones del café" a ser los centros fabriles más importantes del país en ese momento (De Oliveira, 1977; Caldeira, 2007).

El paisaje urbano, por lo tanto, va a sufrir transformaciones radicales que tendrán un impacto directo en los procesos de planeación y generación de lo políticas. Así, de la mano de la construcción de grandes proyectos habitacionales para los obreros, se van a hacer evidentes las necesidades de dotación de servicios urbanos: saneamiento, electrificación y transporte público, a lo que hay que agregar, por otro lado, la infraestructura de salud, educación y recreación. En el mismo periodo se pondrán en funcionamiento, asimismo, proyectos viales y de transporte de gran envergadura: circuitos viales, anillos periféricos y la construcción de sistemas de transporte férreos (metro).

No obstante la construcción de obras de infraestructura de gran impacto y la fluidez de recursos económicos para inversiones urbanas, el panorama de las ciudades tendía, como consecuencia de su desbordado y poco organizado crecimiento, a la fragmentación, con lo cual los fenómenos de exclusión y segregación se hacían más palpables. Aunque éstos últimos fenómenos 
no eran nuevos, sí se vieron favorecidos por el incremento del desempleo que se tradujo, como era de esperarse, en la aparición de una economía de subempleo muy potente que no fue atendida de forma decidida por los gobiernos (Galeano y Beltrán, 2008). Este subempleo no solo representó para los sectores más pobres una posibilidad de acceso a los medios de subsistencia básicos, sino que constituyó en un factor de modificación de patrones de uso y ocupación del espacio, no solo en las zonas periféricas sino también las más céntricas, en donde se pueden apreciar fenómenos como el aumento del comercio ambulante que ocupa las calles y plazoletas, y la precarización de la vivienda en los mismos centros históricos, que si bien tiene precedentes en procesos urbanos anteriores, se agrava en este periodo (Giglia, 2010; Jaramillo, 2012; Davis, 1999).

Los gobiernos, teniendo en cuenta lo anterior, se enfrentaron al reto de generar políticas urbanas que pudieran satisfacer demandas de grupos sociales diversos y contribuyeran al mismo tiempo a gestionar de manera más adecuada las necesidades de las grandes áreas metropolitanas que empezaban a resentir los efectos del creciente tráfico vehicular, la contaminación y la fragmentación espacial (Islas, 2000; Navarro, 2005). Sin embargo, ya era posible apreciar una espiral de problemas que, por la misma naturaleza de la planeación del momento, eran tratados de manera separada sin contar con una visión integrada de la realidad urbana; esto debido, en parte, a la fuerte centralización política característica de las naciones latinoamericanas.

Otro factor que va a impactar de manera decisiva en lo que aquí hemos denominado crisis urbana, es el de la economía. De esta manera, con una captación de recursos que dependía en gran medida de los "buenos precios de materias primas (de hidrocarburos y productos mineros, más que agrícolas) y condiciones de financiamiento externo excepcionales" (Ocampo, 2007, p. 7), las ciudades no tardaron en resentir los estragos de la crisis de la deuda externa (préstamos con los que se apalancó el proceso de industrialización) y la disminución de la cotización de las materias primas en el mercado internacional. A lo anterior se debe agregar que el sector industrial mostraba ya signos evidentes de agotamiento dado, entre otras razones, por la pérdida de ventajas competitivas de los bienes de consumo nacionales frente a la creciente oferta de productos manufacturados foráneos (Almandoz, 2008).

Así, con el advenimiento de la crisis de la deuda externa a principios de la década del 80, las cifras de desempeño económico para América Latina no eran alentadoras, lo cual se puede ver en la caída general del PIB que pasó de 4,5\% en los setenta a 1,3\% en la década siguiente (BulmerThomas, 2010). Para el caso de México, el PIB pasó de crecer con un promedio anual del 6,05\% en 
el tiempo del llamado milagro mexicano -política de desarrollo estabilizador-al 0,18\% después de la crisis, y solo mostrará signos de mejoría hasta 1994 con un 3,91\% (CEFP, 2003).

El aumento de los intereses de los créditos contraídos para financiar los programas de industrialización, el alto gasto público en programas de bienestar, las tasas de recaudación fiscal bajas como consecuencia del estancamiento general de la economía y el evidente deterioro ambiental causado por décadas de uso intensivo de recursos sin adecuados programas de mitigación, son algunos avisos de alerta que prendieron las alarmas tanto para los gobiernos como para los acreedores internacionales.

"Latinoamérica exhibía así los efectos más dramáticos de la sobre urbanización, tales como la hipertrofia del sector terciario y de la economía informal que camuflaba el excedente de fuerza de trabajo en las ciudades, completada con la proliferación de asentamientos no controlados y pobreza" (Almandoz, 2008, p. 69). La respuesta a todo ello trajo consigo un imperativo representado por la necesidad de lograr el equilibrio entre tres variables, que luego van a convertirse en el fundamento de los programas de desarrollo urbano sostenible. Por un lado, las económicas: los ingresos generados por las diversas actividades productivas, los programas de inversión en obras públicas y los pagos del servicio de la deuda. De otro lado, las sociales: mitigar el impacto del evidente estancamiento de la industria en la pérdida de empleos y el consecuente aumento del descontento en las capas más bajas de las sociedades urbanas. Por último, las ambientales: reducción de las emisiones de gases a la atmósfera en el caso de las ciudades, y control a la explotación de recursos en áreas protegidas.

Estos rasgos generales de la crisis urbana de la década del ochenta, que se extenderá hasta finales del siglo pasado, vinieron acompañados por nuevas necesidades que surgen a la par con los desequilibrios económicos del momento $-y$ tal vez por ellos mismos-. Enunciamos enseguida tres de las necesidades que tuvieron un importante eco en los cambios que se van a dar en el futuro, de los cuales, los sistemas BRT son uno de sus resultados más evidentes.

1. Amplios sectores de la sociedad que piden una mayor participación en gestión de la ciudad, más allá de los meros instrumentos políticos formales que en muchos casos ya existían;

2. organismos internacionales que como parte de los paquetes de rescate económico Programas de Ajuste Estructural del FMI, en algunos casos-, buscan que se adelanten reformas a la organización de los estados, en las que haya una mayor participación de la iniciativa privada en la prestación de servicios antes confiados únicamente a la esfera 
pública, así como mejores condiciones institucionales que favorezcan la descentralización política;

3. grupos empresariales que persiguen el tránsito de la inversión centrada en la industria a una orientada a los servicios, para lo cual es necesario contar con infraestructura que lo permita y en la cual están decididos a contribuir para su realización.

Finalmente, es pertinente acotar que los rasgos de la crisis urbana atrás descritos no se presentaron de manera uniforme en el amplio espectro de las ciudades latinoamericanas. Por el contrario, existieron factores locales o endógenos que configuraron la realidad urbana de formas muy diversas. Éstos fueron alentados por las tasas de industrialización precedentes, los niveles de urbanización y expansión de la mancha urbana o por la presencia que tuvo el estado en áreas estratégicas y el papel que éste jugó en los nuevos procesos económicos, políticos y sociales derivados de la aplicación de políticas de liberalización de mercados, desregularización y descentralización.

\section{Los cuatro elementos discursivos del BRT}

La demanda de modelos de movilidad sostenible

La oferta de transporte público en la ciudad de México estuvo marcada en la segunda mitad del siglo pasado por los siguientes procesos: a. la desaparición progresiva de los tranvías (Leidenberger, 2011); b. la consolidación de la Alianza de Camioneros, no solo como prestador del servicio del transporte sino como actor político en la esfera local y nacional (López Saldívar, 1999); c. la construcción del Sistema de Transporte Colectivo - Metro y; d. la conformación de la empresa Ruta 100 por parte del Gobierno del Distrito Federal (GDF) (Davis, 1999).

Cada uno de los modelos de oferta de transporte tuvieron esquemas de gestión particulares que se vinculaban tanto con parámetros propios del sector, como con relaciones políticas que se tejieron en el tiempo, y que tienen sus raíces en procesos que Diane Davis (1999) ha ubicado en la construcción del estado mexicano posrevolucionario. De tal suerte, la llegada a la ciudad de México del Metrobús -con su aparato de gestión propio- representó un cambio no solo en la estructura de las vialidades intervenidas sino también en la concepción más amplia de la movilidad urbana.

Los programas de movilidad sostenible, en los que se enmarca el Metrobús, surgen en el contexto de los proyectos de desarrollo urbano sostenible (DUS) que de acuerdo con Víctor Urquidi 
(2002) "tienen su origen en los años setenta y ochenta, cuando se empezó a advertir y alertar acerca de los peligros de los excesos de contaminación" (p. 526) que estuvieron alentados en América Latina, en gran medida, por el modelo de desarrollista al que ya nos referimos.

Por otra parte, en la evolución del paradigma de sostenibilidad, el informe Nuestro futuro común (también conocido como Informe Brundtland) publicado en 1987, y que es producto de las reuniones de la Comisión Mundial sobre Medio Ambiente y Desarrollo, se va a convertir en un documento fundamental para pensar los desafíos de un desarrollo no solo centrado en factores económicos, sino que considere de forma decidida tanto el cuidado del medio ambiente como la atención de demandas sociales. Por ello la cumbre de Río de 1992 retomará el concepto de desarrollo sostenible y lo pondrá como el eje central de su declaración final, la Agenda 21, suscrita por los delegados de los países reconocidos por la ONU.

Como producto de las discusiones sobre un programa mundial de sostenibilidad, la Agenda 21 propone tres condiciones necesarias para cumplir con las metas de desarrollo sostenible en las ciudades, y que Boris Graizbord (2011) ha sintetizado en las siguientes líneas:

1. Nivel adecuado para todos, de tal forma que por lo menos logren estándares mínimos en la vivienda, la infraestructura, la seguridad social y la estabilidad

2. Modos de producción y consumo sostenibles; y

3. Forma y estructura urbanas que promuevan o faciliten las condiciones propuestas en 1 y 2 . (p. 42)

En este orden de ideas, los dos documentos referidos -el informe Nuestro futuro común y la Agenda 21- como pilares del desarrollo urbano sostenible, abordan la necesidad de pensar modelos de transporte que contribuyan no solo a la reducción de las emisiones de gases contaminantes a la atmósfera, sino también a la mejoría de la calidad de vida de sus usuarios condiciones de calidad, seguridad y accesibilidad-.

De allí que la movilidad sostenible a la que se alude haya sido definida por un sinnúmero de instituciones tanto nacionales como internacionales. Para nuestro caso, vamos a tomar la que de ella hacen David Guillamón y David Hoyos (2005) en su libro Movilidad sostenible. De la teoría a la práctica:

"un sistema y unas pautas de desplazamiento que proporcionan los medios y las oportunidades para satisfacer las necesidades económicas, ambientales y sociales de manera eficiente y equitativa, al mismo tiempo que minimiza los impactos adversos evitables o innecesarios y sus costes asociados, en escalas espaciales y temporales relevantes" (p. 11). 
No se trata, por lo tanto, de una cuestión meramente ambiental lo que persigue el paradigma de la sostenibilidad, sino que su campo de acción se amplía al ámbito económico y social. Además, al hablar de los "impactos adversos evitables" (Guillamón y Hoyos, 2005, p. 11), se trata de poner de presente lo que Lucy Winchester (2006) ha denominado "equidad intergeneracional" (p. 9), que no es más que la posibilidad que se le brinda a futuras generaciones de gozar de "una cantidad de bienes al menos iguales a los que disponemos hoy" (p. 9).

Igualmente, Guillamón y Hoyos (2005) exponen la definición que la Organización Económica para la Cooperación y el Desarrollo (OECD, por sus siglas en inglés) - de la cual hace parte México- ofrece sobre movilidad sostenible, y aunque el énfasis de ésta es diferente a la de la Comisión Europea, es indiscutible que tienen puntos comunes que las ligan con las conceptualizaciones que al respecto se han hecho:

un sistema de transportes sostenible es aquel que "sin dañar la salud pública ni los ecosistemas, satisface la necesidad de acceso de acuerdo con el uso de recursos renovables por debajo de su tasa de regeneración y el uso de recursos no renovables por debajo de la tasa de desarrollo de substitutos renovables" (p. 12).

En la postura de la OECD se puede apreciar, en consecuencia, la preocupación por estructurar salidas a la crisis ambiental que, en el marco de la sostenibilidad, permitan el desarrollo de tecnologías limpias que no interfieran con el ciclo de recuperación de recursos renovables; pero además, teniendo presente en el horizonte de acción, que el desarrollo de sustitutos renovables tiene procesos de generación de largo plazo, dados los exhaustivos procesos de investigación que esto conlleva.

En este punto de la discusión, convendría ver la manera en la que los sistemas BRT responden a las expectativas de la movilidad sostenible. Para ello, revisaremos dos documentos vinculados con la implementación de los mismos en diferentes ciudades de América Latina, y que a nuestro juicio pueden ilustrar de manera clara el enfoque sostenible de las propuestas en mención.

EI PNUMA, Programa de las Naciones Unidas para el Medio Ambiente (UNEP, por sus siglas en inglés), se ha convertido, después de la experiencia del Transmilenio en Bogotá, en un importante promotor de los sistemas BRT en América Latina. Es así como ha desarrollado y publicado a lo largo de la década pasada, en asocio con instituciones encargadas de la investigación y consultoría en temas vinculados con el transporte (ITDP, WRI, RCSC y CTS) ${ }^{4}$, documentos que buscan guiar la implementación del BRT en la región y en el mundo.

\footnotetext{
${ }^{4}$ Institute for Transportation and Development Policy, World Resources Institute, Ross Center for Sustainable Cities y Centro de Transporte Sustentable.
} 
En primer lugar, el documento Planificación e implementación de un sistema de Bus Rápido en América Latina: resumen orientado a tomadores de decisiones (Rogat, 2009), se refiere a la movilidad sostenible en los siguientes términos:

"un sistema de transporte público de una ciudad es un componente fundamental de una política de transporte sostenible orientada al beneficio de sus usuarios y pensando en los beneficios a largo plazo para la ciudad. Un aspecto que se toma en cuenta crecientemente al tomar decisiones sobre un sistema de transporte son las implicaciones en términos de desarrollo sostenible y la relación que éste tiene con uno u otro proyecto de transporte. El desarrollo sostenible ha sido un concepto con varias décadas de desarrollo, cuyos componentes principales son los económicos, ambientales y sociales" $(p, 8)$.

Como se puede apreciar en el anterior apartado, y retomando las definiciones hechas por la Comisión Europea y la OCDE, es posible encontrar elementos transversales que las ligan con la conceptualización que sobre el desarrollo sostenible que ha expuesto, tales como la cuestión multifactorial, no solo ambiental, de las propuestas de Desarrollo Urbano Sostenible.

Con respecto a la dimensión ambiental del desarrollo sostenible, el texto de Rogat (2009) al momento de describir las características técnicas de las unidades BRT, pone un énfasis particular en la reducción de las emisiones de éstas con respectos a las de los vehículos particulares y las demás opciones de transporte público automotor - se excluye por tanto al metro, debido a que no usa combustibles fósiles sino corriente eléctrica para su funcionamiento.

En cuanto a los otros dos aspectos funcionales del desarrollo sostenible - el económico y el social -, el texto de Rogat (2009) resalta que los sistemas BRT no solo involucran medidas para reducir las emisiones de gases contaminantes, sino que lo que lo que hay detrás de estos proyectos constituye, de entrada, un programa amplio de organización de la ciudad. Así, "los proyectos que den mayor prelación al transporte público, las bicicletas y caminar tendrán efectos más benéficos para la población" (p. 10), y estos permiten, en la misma línea, la reorganización del espacio público intervenido y la revitalización de zonas deprimidas. Empero, es pertinente acotar que en su momento otros modelos de transporte como el tranvía o el metro, también planteaban que su puesta en marcha contribuía no solo a mejorar la movilidad en las zonas urbanas sino que representaban cambios importantes en cuanto al cambio en patrones de uso del suelo, revitalización de áreas deterioradas y reordenamiento general del espacio público.

En igual sentido, las variables económicas son tomadas en consideración, toda vez que "la eficiencia de una ciudad se refleja en la facilidad de movimiento a lo largo de la misma" (Rogat, 
2009 , p. 9) y, por lo tanto, la reducción de los tiempos de traslado entre las zonas de residencia, trabajo, estudio y esparcimiento, traerá consigo "una mayor eficiencia económica" (p. 9).

En segundo lugar, el PNUMA, en asocio con diversas instituciones, entre las cuales cabe resaltar al ITPD por su papel en la promoción del BRT en el continente, publica la Guía de planificación de los sistemas BRT. Autobuses de Transito Rápido (ITDP, 2010). Este documento, que tiene un mayor nivel de detalle que el anterior, centra su atención en las potencialidades del modelo BRT frente otras propuestas de transporte como el tranvía, el tren ligero o el metro, aunque no las descarta como soluciones efectivas.

El documento resalta que "todas las opciones de transporte público producen impactos ambientales" (ITDP, 2010, p. 94) dados, fundamentalmente por el tipo de combustibles utilizado y también por la posibilidad de reducir, vía la oferta de calidad, el porcentaje de viajes en vehículos particulares. No se trata, según se puede concluir de los postulados del documento, de la eliminación de las emisiones, pues aunque las unidades también podrían ser tipo trolebús, como en el caso de Quito, en la mayoría de los casos emplean "diesel limpio" o gas natural que contribuyen a la cuota de gases de efecto invernadero.

Ahora bien, lo que se propone como verdadero aporte de estos sistemas a la reducción de los impactos ambientales negativos, es la posibilidad de reemplazar las unidades más contaminantes, dado sus años de uso y su tecnología obsoleta, por unas de mayor capacidad, bien sean articuladas o biarticuladas, que cuentan con motores de tecnologías más avanzadas. "Dado que un vehículo de transporte público es igual a 100 veces o más vehículos individuales (...), la reducción en emisiones al aire pueden ser considerables si la demanda se incrementa" (ITDP, 2010, p. 95), y así, a la par con el beneficio ambiental, también es posible lograr una mayor eficiencia en la gestión de los viajes.

De otro lado, el documento refiere las bondades del sistema en cuanto a: la mejora en la calidad de vida de los usuarios; la disminución de los tiempos de desplazamiento; la generación de empleos más estables para los operadores del sistema de carriles confinados, dado el nuevo esquema de funcionamiento que, al ser de corte empresarial, tendrá un lógica laboral que se aparta diametralmente del modelo hombre-camión tradicional; mejores condiciones de accesibilidad para personas con capacidades especiales. En síntesis,

"los sistemas de transporte público también pueden proporcionar uno de los pocos lugares en una ciudad donde todos los grupos sociales pueden encontrarse e interactuar. (...) La regeneración de un área urbana gracias a las mejoras en el transporte público puede tener beneficios sociales

Revista de Direito da Cidade, vol. 09, no 1. ISSN 2317-7721 pp. 136-162 145 
múltiples. Como fue anotado, la mejora de un área crea empleo y crecimiento económico" (ITDP, 2010, p. 96).

Así, de acuerdo con estos dos documentos revisados, los sistemas BRT contribuyen de manera decisiva al desarrollo urbano sostenible en sus tres ámbitos de acción. Sin embargo, es pertinente anotar que las condiciones de implementación de esta alternativa de transporte en las distintas ciudades, obedecen a criterios y condiciones particulares de organización del espacio urbano, por lo cual es posible que en algunos casos no se logren desarrollar plenamente las potencialidades de los carriles confinados.

Las iniciativas de inversión mixtas (private-public partnership)

El segundo elemento del aparato discursivo del BRT está dado por la promoción de las iniciativas de inversión mixtas (público-privado) en la prestación de servicios urbanos, en particular el transporte. En este apartado revisaremos la forma en la que el sistema de carriles confinados apropia este mecanismo de gestión para aplicarlo en su estructura o plan de negocio (ITDP, 2010).

La aparición de nuevos agentes y organizaciones al interior de las ciudades en el periodo de crisis urbana, es el signos más visible del "cambio institucional" (North, 1995) que opera en la gestión urbana a partir de la década del ochenta. Lo anterior implica que las redes de actores que se generan allí, van a estar medidas por intercambios de información y conocimientos que tienen como sustento un nuevo marco político que sirve, a su vez, como instrumento regulador de las acciones emprendidas.

De igual modo, la misma incorporación de términos como gobernanza y nueva gestión urbana, (Aguilar Villanueva, 2006; Alfie, 2009; Seixas, 2008; Delgado, 2009) va a significar, más que una mera transformación semántica, una ruptura con una tradición regulatoria que provenía del mismo agente que ejecutaba los proyectos (el Estado). La gobernanza urbana y la nueva gestión urbana, por tanto, van a centrarse en la posibilidad de vincular la redistribución de poder -que es la esencia de la descentralización al entender de Sergio Boisier (2001)-, la participación ciudadana con facultades de decisión y no únicamente de consulta u opinión, y la capacidad de organismos privados de diversa naturaleza, en la prestación de servicios urbanos que antes estaba restringida al estado.

En la nueva "arquitectura" de la política y planeación de las ciudades, sostiene Eduardo Passalacqua (2003), los gobiernos deben fungir como "agentes-promotores" (p. 7), de los proyectos urbanos, en los cuales el comportamiento que asuman sea el de "un facilitador de oportunidades más que distribuidor de recursos públicos" (p. 7). Sin embargo, eso no se logrará 
con la simple reasignación de funciones, por lo que se hace necesario que se desarrollen marcos regulatorios y normativos que permitan, tanto a las instancias de gobiernos como los nuevos actores desempeñar sus papeles en la escena urbana.

Conviene en este punto acotar que estas tendencias de gestión que fueron arribando a Latinoamérica desde la década del ochenta estaban siendo alentadas por procesos políticos de alcance mundial. Partiendo principalmente de Estados Unidos con el gobierno de Reagan e Inglaterra con el de Thatcher, se estaba produciendo un giro cardinal en cuanto a las implicaciones de tener un estado que interviniera en todos los ámbitos de la sociedad, máxime cuando las constantes crisis económicas estaban minando los índices de recaudación fiscal con la cual se sostenían los programas de bienestar social.

Estas tendencias mundiales de desplazamiento del papel del estado en cuanto a la prestación de servicios públicos, como lo acota Passalacqua (2003), tuvieron en el continente formas particulares de implantación, dadas las condiciones políticas y económicas de cada nación. Asimismo, las consecuencias de procesos de apertura de estos servicios al capital privado sin contar con instrumentos claros de regulación, conllevaron, en algunos casos, implementación de políticas de precios restrictivas con las cuales comunidades con menores ingresos vieron impedido el acceso a los mismos, con lo cual se agudizaron las condiciones de desigualdad al interior de las ciudades (Burgess, 2003; Balbo, 2003).

La convicción -heredada en gran medida del nuevo marco de gestión- de que la prestación de servicios urbanos, particularmente el transporte, tendría mejores índices de calidad y eficiencia al ser asumida por actores privados en asocio con los gobiernos, es retomada por las instituciones dedicadas a la promoción de los sistemas BRT. Así pues, la tendencia de asociación público-privada en el caso de los carriles confinados va a estar presente en las agendas gubernamentales de las principales ciudades latinoamericanas, independientemente, incluso, de la orientación política de las administraciones locales. Ese es justamente el sentido que tienen las tendencias y fenómenos urbanos que estamos tratando de describir: las urbes no pueden sustraerse de ellos, pues aún cuando generen o haya manifestaciones específicas que estén encaminadas a adaptarlos a sus contextos locales, esta serie de parámetros de gestión circulan de manera global.

Antes de describir las características del modelo de negocio propuesto en la Guía de planificación de los sistemas BRT. Autobuses de Transito Rápido (2010), conviene decir que la forma tradicional de operación del sistema de transporte público concesionado está basada en permisos de circulación por determinadas vialidades (rutas) o en concesiones a empresas particulares, sobre 
las cuales las autoridades públicas encargadas de la administración o vigilancia del servicio han manifestado bajos niveles de control (Ver esquema 1). De lo anterior se excluyen, por su misma naturaleza, las empresas públicas dedicadas a la prestación de transporte automotor.

Esquema 1: Funcionamiento del sistema de transporte público concesionado

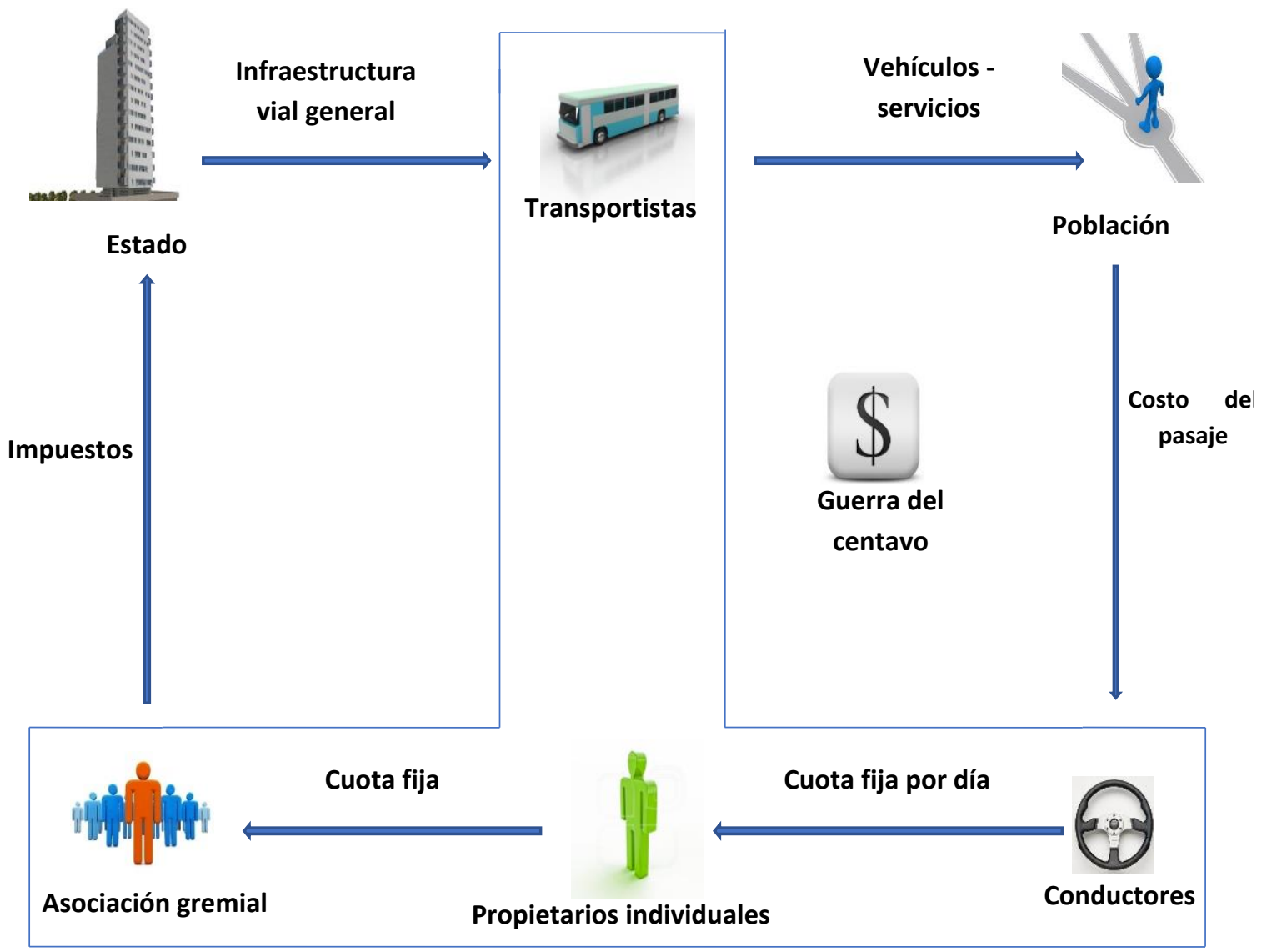

Fuente: Modificación parcial del modelo que aparece en el documento: (CTS, 2005)

Como se puede apreciar, el punto central de esta matriz de operación es la "guerra por el centavo", característica de un esquema de funcionamiento centrado en la competencia desregulada, en la cual cada conductor de unidad -hombre-camión- debe asegurar un número mínimo de pasajeros transportados como condición salarial básica (Iracheta, 2005; Hernández, 2005; Salazar, 2008). Lo anterior tiene un impacto negativo no solo en la calidad del servicio sino también en la movilidad de las ciudades, si se tiene en cuenta que la baja capacidad de las unidades en servicio y la permisividad en cuanto a las paradas aleatorias congestionan las vialidades, generando de esta forma traumatismos en el tráfico, con lo cual los tiempos de traslado se hacen cada vez más elevados. 
Como señala Alfonso Iracheta (2005), los directos afectados de este esquema de negocio son los usuarios, quienes diariamente deben acudir al transporte tradicional para lograr desplazarse por la ciudad. Sin embargo, no debe dejarse de lado el impacto negativo en el desempeño económico de las urbes: la falta de conectividad, la imposibilidad de integrar redes de transporte, las tarifas asociadas a la necesidad de abordar diversos medios de transporte en un mismo trayecto, hacen que uno de los factores que se consideran decisivos a la hora de evaluar el desarrollo urbano, como es el de la movilidad, muestre cifras negativas.

En este orden de ideas, generar una nueva "estructura de negocio" (ITDP, 2010, p. 8) se convirtió en un imperativo de la modernización del transporte público en las principales ciudades de América Latina. Así, la Guía de planificación de los sistemas BRT. Autobuses de Transito Rápido (2010, p. 601), propone cinco elementos necesarios para lograr una estructura organizativa acorde con las expectativas tanto de los usuarios como de los inversores del sistema:

1. Ambiente institucional de regulación en el cual las firmas privadas de concesión operen el sistema con amplia veeduría pública.

2. Logro de comportamiento de costos en marco de sociedades públicas-privadas (p. ej., el sector privado financia los vehículos).

3. Proceso de licitación de operadores que fomente la competencia para el mercado pero limite la competencia al interior del mercado.

4. Compensación de operadores basada en kilómetros-vehículo recorridos en vez de número de pasajeros.

5. Sistemas de recolección de tarifas con concesión independiente que distribuyan las ganancias de forma totalmente transparente.

"Este sistema mixto de regulación pública y operación privada se ve como la aproximación óptima para lograr un sistema competitivo y transparente que responda a las necesidades del usuario" (ITDP, 2010, p. 601). Es claro en este sistema de funcionamiento que así como se generan nuevas relaciones contractuales entre el gobierno y los prestadores del servicio, también emergen nuevas relaciones políticas entre estos actores, basadas fundamentalmente en los postulados de la gobernanza urbana.

En estos términos, el BRT, al oponerse al modelo hombre-camión, impone, a su vez, sus propios criterios de negociación que obedecen a los nuevos arreglos institucionales expuestos, en los cuales las organizaciones tradicionales de transportistas -asociaciones, alianzas o sindicatos- se 
ven en la necesidad de migrar hacia formas empresariales para lograr insertarse en el nuevo modelo de negocio so pena de quedar excluidos.

En su libro El leviatán urbano, Diane E. Davis (1999) va a describir de forma detallada el papel que jugó el transporte público en la configuración espacial de la ciudad de México en el siglo pasado. Centrando su atención en las relaciones entre los prestadores de este servicio y las instancias de gobierno, la autora delinea un mapa político ${ }^{5}$ que fue decisivo en el proceso de desaparición del tranvía, en la construcción del Metro, en la consolidación del llamado "pulpo camionero" ${ }^{\prime 6}$ y, por último, en la constitución de la empresa pública Ruta 100, que surge en 1981 como solución al excesivo poder manifestado por el sector de los transportistas permisionarios.

La anterior referencia es válida para remarcar el hecho de que la construcción del BRT no solo obedece a criterios urbanísticos sino, como lo menciona la Guía de planificación de los sistemas BRT. Autobuses de Transito Rápido (ITDP, 2010), a decisiones políticas que se muestran como obligadas para lograr los fines propuestos, y que están encaminados a lograr vincular la prestación del servicio de transporte público con criterios de corte neoliberal dados en el marco de la crisis urbana ya descrita. Al respecto, es revelador el apartado dedicado en el documento en mención a la "transformación de los sistemas existentes" (ITDP, 2010, p. 601). Primero habla del escenario de creación de un sistema BRT a partir de un monopolio estatal, y segundo, siendo este el punto que nos interesa resaltar acá, de la "transformación de una industria de autobús privada, débilmente regulada e informal" (ITDP, 2010, p. 607).

Con relación a esta última transformación, el documento expone un plan de acción para las negociaciones en el caso del Transmilenio en Bogotá:

El Alcalde se reunió primero con los jefes de las empresas de buses y les dijo que el BRT comenzaría a andar con o sin ellos; que ellos podrían o bien participar de forma productiva o bien perder sus derechos de operación en rutas de TransMilenio. Después de esto, nadie de la oficina del Alcalde se reunió con los operadores privados (...) Si los operadores privados de buses ya tienen una concesión de 15 años para operar servicios exclusivos de buses, el operador privado de buses podría frenar el proyecto BRT en las cortes, durante años. En esta situación el gobierno deberá "comprar" al

\footnotetext{
${ }^{5}$ Este mapa político estuvo determinado, en gran medida, por la presencia, en el panorama del transporte público, de organizaciones sectoriales afiliadas a corporaciones políticas del PRI como la Confederación Nacional de Organizaciones Populares - CNOP, la Confederación Regional Obrera Mexicana - CROM o la Confederación de Trabajadores de México - CTM. De esta forma, en el complejo sistema corporativista, la participación protagónica de la Alianza de Camioneros de México, influyó, de acuerdo con Davis, en la negativa del Regente Ernesto Uruchurtu al proyecto de ICA para la construcción del tren subterráneo en la ciudad.

6 Denominación que se le ha dado al periodo de expansión descontrolada del transporte público concesionado entre 1946 y 1981 (Voukas y López, 2011).
} 
operador. Sin embargo, normalmente lo operadores de buses son culpables de centenares de pequeñas violaciones reguladoras y estas violaciones se pueden utilizar para presionar a los operadores a sentarse en la mesa de negociación" (ITDP, 2010, p. 610).

Como se aprecia en la anterior cita, dejando de lado los mecanismos empleados para lograr un acuerdo, esta tendencia de asociación público-privada no está exenta de problemas para su consecución, dado que el proceso de cambio institucional se encuentra atravesado por inercias organizativas presentes en el gremio de los transportadores, puestas en términos principalmente de la desconfianza (Salazar, 2008), que data de varias décadas atrás, y que se manifestarán, claramente, en todo el conflicto generado por la construcción de los carriles confinados de transporte.

El impacto del fenómeno de la globalización en la gestión de las ciudades

El tercer ámbito de análisis de la implementación del BRT está dado por el impacto del fenómeno de la globalización en la gestión de las ciudades. Para abordar este punto nos vamos a referir a dos aspectos que han contribuido a la expansión del modelo de carriles confinados en América Latina. El primer aspecto es el de la circulación de ideas acerca del desarrollo urbano en un contexto globalizador. El segundo aspecto, referido al papel de las corporaciones en la promoción del sistema de carriles confinados en la región, si bien no se hace explícito en los dos documentos revisados (Guía de planificación de los sistemas BRT. Autobuses de Transito Rápido y Planificación e implementación de un sistema de Bus Rápido en América Latina: resumen orientado a tomadores de decisiones), consideramos que gravita sobre estas propuestas.

\section{La circulación de ideas del urbanismo en la globalización}

De acuerdo con Luis Mauricio Cuervo (2005, p. 30), existen tres "canales a través de los cuales se da el proceso global de circulación de ideas de ciudad y de desarrollo urbano: la teoría urbana, los paradigmas urbanos y el discurso institucional público (nacional e internacional)". Cada uno de estos vectores posee características específicas, sin embargo, lo que nos interesa resaltar aquí es que hay un elemento común entre ellos: la información.

Siguiendo a Cuervo, los modelos teóricos "surgen de la observación de experiencias concretas que (...) a través de la elaboración de estudios e investigaciones y de su difusión, terminan convirtiéndose en puntos de referencia obligados y en imágenes del deber ser urbano o territorial" (Cuervo, 2005, p. 27). A su vez, los paradigmas tienen un mecanismo particular de 
acción que es la observación directa de experiencias por parte de diversos sectores de la sociedad como los "funcionarios y políticos comprometidos en la gestión del desarrollo urbano y local", además de "los movimientos sociales organizados, tales como viviendistas, ambientalistas, organizaciones y asociaciones de vecinos" (Cuervo, 2005, p. 27). Por otro lado, el discurso institucional está dado "por instituciones y organizaciones nacionales e internacionales dedicadas a los temas del desarrollo regional y urbano: bancos internacionales de fomento (BM, BID), fondos nacionales especializados (...), instituciones públicas nacionales dedicadas a temas específicos tales como vivienda, transporte, servicios públicos domiciliarios, infraestructura urbana" (Cuervo, 2005, p. 31).

La distinción que hace el autor entre estos tres canales es meramente analítica si se tiene en cuenta que las fronteras entre cada uno de los actores involucrados allí son porosas. Importa, de otro lado, reconocer que la gestión de las ciudades en el contexto de la globalización no es responsabilidad exclusiva de los gobiernos, sino que ahora intervienen en ella una amplia gama de actores, que no manifiestan sus intereses de manera aislada o unitaria. Se generan, de la mano de los flujos de información entre ellos, redes de cooperación que los llevan a integrarse a movimientos ya no de alcance meramente local sino también global (Sassen, 2007).

Lo anterior no quiere decir que antes de la década del setenta u ochenta no hubiera relación entre los centros de investigación ligados a las universidades, los gobiernos y las instituciones internacionales. Lo que cambia es el caudal de información que circula entre ellos, y que tienen como elemento detonante la ampliación de las posibilidades de comunicación en todos los niveles.

Con base en lo que se ha señalado, se pueden observar, en cuanto a la circulación de ideas de ciudad, dos momentos de un mismo proceso: por un lado, se encuentra el reconocimiento y promoción, en el concierto internacional, las capacidades locales de cada ciudad, denominadas por George Benko (2000) "especificidades del lugar", y por otro, se aprecia la incorporación de éstas a "redes globales de decisión e intercambio" que se manifiestan en patrones territoriales, igualmente globales (Borja y Castells, 1999).

Este proceso ejemplifica de forma clara las "tendencias y fenómenos" (De Mattos, 2001) de gestión urbana en la globalización, a los que nos referimos al principio del artículo. Si volvemos sobre los dos documentos diseñados para implementación del BRT, podremos encontrar que allí se hace referencia a las amplias posibilidades que ofrece el sistema de carriles confinados en un contexto global marcado por la necesidad de ordenar el transporte público en función de la 
movilidad, y de que ésta atienda a criterios de sostenibilidad; pero también se resalta que su implementación depende de las características y necesidades específicas de cada ciudad.

El papel de las corporaciones en la promoción de los carriles confinados

En el acápite anterior nos referimos al proceso de integración de los actores involucrados en la gestión urbana en redes globales de cooperación y decisión. Se dijo, además, que los gobiernos, en el marco de las reformas adelantadas como respuesta a la crisis urbana de la década del ochenta y noventa, se vieron abocados a cumplir un papel diferente al que venían cumpliendo hasta ese momento.

La función que históricamente habían representado los gobiernos como arquitectos de las políticas sectoriales sufre profundas transformaciones a raíz de los procesos políticos y económicos que se vivieron en las dos últimas décadas del siglo pasado. Las estructuras de gobierno, en medio de los procesos de descentralización, apertura económica y liberalización de los mercados, actuarán en la nueva realidad urbana como "agentes-promotores" de proyectos de intervención que se encuentran alentados ahora por un sentido de competitividad y captación de recursos e inversiones.

Las ciudades deben mostrar, por lo tanto, resultados de gestión que les permita ubicarse como importantes polos de atracción de inversiones, turistas, servicios, pero además como centros de promoción cultural y artística en un contexto de competencia global (GDF, 2011). Uno de los elementos necesarios para lograrlo, es contar con una infraestructura capaz de servir como soporte de las nuevas necesidades. De esa manera, la movilidad va a ser uno de los elementos más visibles en los programas de desarrollo urbano del presente: desde los planes de recuperación de los centros históricos, hasta las iniciativas metropolitanas y de integración ciudad-región.

Dado el desplazamiento (y contracción) de funciones de los gobiernos, aparecen nuevos actores interesados en el desarrollo urbano: instituciones internacionales dedicadas a consultorías, institutos de encargados de investigaciones sectoriales y, por último corporaciones interesadas en entrar a hacer parte del proceso de planeación. Éstas últimas, se posicionarán progresivamente como actores encargados de gestionar, junto con los gobiernos y la sociedad civil, las ciudades. Su intervención se da tanto en diversos niveles como a través de diferentes organizaciones.

En el caso de los proyectos de movilidad urbana sostenible, y por consiguiente, del BRT, las corporaciones participan creando fundaciones encargadas de apoyar iniciativas locales de reorganización de los servicios urbanos; además financian, a través de las mismas fundaciones o de 
manera directa, estudios sobre el impacto de las emisiones de contaminantes del transporte público en el deterioro ambiental y congresos en los que se aborda la urgencia de adoptar medidas de movilidad sostenible (De la Cruz, 2010) (Volvo, 2010); publican, asimismo, documentos en los que promueven sus productos (autobuses en la mayoría de los casos) como respuesta a los desafíos de sostenibilidad urbana; y se convierten en socios estratégicos de las empresas concesionarias de los sistemas de carriles confinados ${ }^{7}$.

Con base en lo descrito, es posible leer el interés de promover acciones de movilidad sostenible, centradas en el BRT, desde múltiples ópticas. Por parte de los gobiernos, la sociedad civil y algunas instituciones internacionales, hay una necesidad de instrumentar salidas al problema del transporte público, y por parte de las corporaciones se manifiesta un interés por aumentar, de un lado, sus perspectivas de negocio, y de otro, por ganar protagonismo e influencia en la esfera de la planeación urbana.

Transporte público masivo más humano

El cuarto ámbito de análisis del discurso del BRT, está constituido por la llamada humanización del transporte público. Así, los sistemas de tránsito rápido se constituyen, de acuerdo con las entidades promotoras del mismo, en la punta de lanza de una sustancial transformación en la manera de entender el transporte y la movilidad. En primera medida, según Ricardo Montezuma (2010), "uno de los retos del transporte es pasar de la exaltación de la rapidez que ha perseguido durante más de un siglo, a la promoción del disfrute del desplazamiento, a la oferta de convivialidad" (p. 210). En este sentido, toma forma la idea de que la estructura espacial de los sistemas BRT está orientada a lograr una mayor integración entre los pasajeros, al garantizar, por ejemplo, la accesibilidad a personas con capacidades diferentes.

En segundo lugar, y en consonancia con el contenido del desarrollo sostenible, de lo que se trata es de hacer que el Metrobús sea un sistema que además de movilizar pasajeros transforme el rostro de la ciudad y, en última instancia, la experiencia de los usuarios. De tal suerte, la

\footnotetext{
${ }^{7}$ Como muestra de lo anterior, podemos citar el caso de la compañía anglo-neerlandesa Shell, la cual promueve, a través de la Fundación Shell, el desarrollo mundial y, además, le hace frente a "los desafíos medioambientales relacionados con el impacto de la energía y la globalización". No debemos olvidar, sin embargo, que la fuente de recursos económicos de esta compañía es la explotación, distribución y comercialización de hidrocarburos. Podríamos mencionar también a la Fundaciones Caterpillar (dependiente de una importante compañía fabricante de motores diesel) o a Volvo y Mercedez Benz, ambas dedicadas a la producción de autobuses para los sistemas BRT, y quienes patrocinan, de forma activa, la realización de foros, reuniones temáticas y seminarios sobre la importancia de este sistema en el concierto internacional de la movilidad.
} 
humanización se entiende, en el marco del discurso del BRT, como aquel estado en el cual, adicionalmente al cumplimiento de parámetros en la calidad de las operaciones (velocidad, espacio en las unidades, seguridad, etc.), es posible hallar actitudes o "ánimos serenos y procesos de desaceleración" por parte de los pasajeros (Montezuma, 2010, p. 226).

Ahora bien, es preciso decir que en estos asuntos que venimos señalando al respecto de la humanización del transporte público se vinculan, en el marco del BRT, tanto los factores internos dados por su estructura funcional-, como con efectos de éste en la forma urbana y en la manera de vivir la ciudad. Es decir, los beneficios de este sistema se pueden apreciar en la mejora de los espacios y las condiciones para el abordaje y descenso de las unidades, y también en las intervenciones en el paisaje urbano, puestas en términos de arborización, adecuación de banquetas $y$, en casos como el de Bogotá, en la construcción de plazoletas y lugares de esparcimiento, obras que el Transmilenio contribuyó a potenciar.

Por otra parte, como lo acota el documento Metrobús: una fórmula ganadora (CTS, 2009), en una ciudad en la que circulan diariamente 3.260.919 millones de vehículos entre particulares, transporte público y de carga, de los cuales el 5\% son de transporte público (EOD, 2007), las emisiones contaminantes se convierten en un factor que genera "más de 4.000 muertes al año (...) causadas por problemas respiratorios acreditados a la calidad del aire" (CTS, 2009, p. 63). En consecuencia, el BRT en la misma medida en la que contribuye a minimizar impactos negativos en el ambiente, también permite aumentar la actividad física de los usuarios, quienes deben caminar "desde los puntos de origen y a los puntos destino" (p. 65) para abordar las unidades.

Humanizar el transporte público es una tarea que el BRT se ha arrogado, y en ciudades latinoamericanas en las cuales no existen otros sistemas de transporte masivo, es natural que el modelo de carril confinado sea tomado como una solución efectiva y de bajo costo para aliviar los problemas de movilización cotidiana de pasajeros (Pardo, 2009). En este punto convendría, pues, mencionar las principales acciones que se emprenden, por parte de los organismos encargados de administrar los sistemas de tránsito rápido, para lograr el efecto de convivialidad y disfrute del transporte público:

1. En diferentes latitudes, los organismos encargados de administrar los sistemas de transporte masivo han advertido la posibilidad de convertir las estaciones, rampas de acceso y los vehículos o trenes en escenarios de manifestación cultural. Así, por medio de exposiciones itinerantes, murales, conciertos y hasta performance, se han explorado las amplias posibilidades de emplear los grises e inertes espacios del transporte como 
mecanismos para acercar a los usuarios a nuevas formas de apropiarse de los espacios urbanos. Todos estos programas han estado orientados, además, por un sentido de lo que podríamos llamar un arte cooperativo, en el que puede intervenir el amplio de la población en la construcción de espacialidades; en últimas, se trata de generar lugares de encuentro y socialización.

Es posible hablar, a este respecto, de experiencias como las del Transmilenio en Bogotá (un sistema de tipo BRT), en donde a través de la campaña Libro al viento este sistema de transporte masivo se convirtió en un escenario propicio de fomento de la lectura. Allí, se dispusieron en las principales estaciones estantes con libros de pequeño formato que incluían antologías de poesía colombiana, cuentos y crónicas sobre Bogotá, y selecciones de obras de literatura universal. Los pasajeros, al acceder a la estación, tomaban alguno de estos tomos, los leían en su recorrido o lo podían llevar a sus casas, con la responsabilidad ciudadana de regresarlos al terminar su lectura, para que de esta forma circulara de "mano en mano" este saber.

Una experiencia similar se pudo advertir en el Metro de la ciudad de México, con el proyecto Para leer de boleto en el metro, que bajo un sistema similar al de Transmilenio, procuraba fomentar el hábito de lectura entre los usuarios de este sistema de transporte. Además, en el mismo Metro, de acuerdo con Olivia Domínguez (2012) y las autoridades del STC-Metro, hay "un programa amplio de actividades relacionadas con la difusión de la cultura y de la ciencia que no se compara con ningún otro espacio de la ciudad de México" (p. 269).

De la misma manera, podríamos hablar de la experiencia del Transantiago en Chile en donde se implementó el programa Santiago en 100 palabras, que buscaba vincular la experiencia cotidiana de los usuarios de este sistema con la organización del mismo, o el del ampliamente conocido metro de la ciudad de Moscú, que aunque diferente a los anteriores, por cuanto su concepto artístico está dado desde el mismo momento de su construcción, sirve para ilustrar el carácter de espacio de encuentro y apreciación artística que pueden tener los sistemas de transporte.

2. Una preocupación que se ha venido convirtiendo en imperativo a la hora de planear y ejecutar proyectos de transporte masivo, es la de la accesibilidad. Este aspecto se encuentra relacionado con uno de los elementos de la sostenibilidad que fueron abordados en otro apartado del presente capítulo: el aspecto social. ¿Cómo lograr que las personas con capacidades diferentes puedan acceder en igualdad de condiciones a los vehículos de transporte, y por extensión, a los espacios urbanos? Los recursos que se han 
dispuesto para tal fin, están dados por el diseño funcional de las estaciones, las zonas de integración y los vehículos del BRT.

Ahora bien, la accesibilidad aparece en el discurso del BRT vinculada, casi en exclusiva, con la posibilidad de brindar a los pasajeros espacios adecuados para abordar, en igualdad de condiciones, los autobuses articulados. Sin embargo, esto desconoce el sentido más amplio de la accesibilidad, el cual encarna la articulación de los bienes y servicios urbanos con la estructura de la movilidad en las ciudades. En otros términos, se trata de lograr que el transporte público funja como un mecanismo de integración de los pobladores tanto al interior de los sistemas, como en lo que respecta a la generación de nuevas centralidades o lugares de actividad.

3. Un tercer elemento que contribuye a hablar de la humanización del transporte público, está dado por la integración modal propiciada por la implementación del BRT. En las ciudades latinoamericanas es común ver que los viajes diarios se realizan en diferentes modos de transporte; para la Zona Metropolitana del Valle de México (ZMVM) encontramos que la composición de los viajes, según la EOD-2007, es la siguiente: 54.9\% son realizados en un único modo - poco más de 8 millones de viajes - y $45.1 \%$ se dan en dos o más modos - de 6.7 millones -.

De la anterior información se desprende la importancia de generar procesos de integración modal, toda vez que la actual estructura de transporte limita la posibilidad de los usuarios para estacionar sus bicicletas en las estaciones del Metro, el Metrobús o el Tren Ligero, situación que impone, de entrada, restricciones para emplear mecanismos alternativos $y$, entre otras cosas, menos contaminantes para movilizarse por la ciudad. Por ello, una de las propuestas que se hacen más presentes en el discurso del BRT es el de lograr articular la oferta de transporte en lo que se refiere a la cuestión tarifaria y de mecanismos de pago -como la tarjeta DF, implementada a finales del año 2012 por el GDF-, y en lo tocante, como se dijo, a la integración física de los modos de transporte.

\section{CONCLUSIONES}

El acercamiento a los elementos que configuran el aparato discursivo del BRT permite comprender la atmósfera del momento en el que los gobiernos locales deciden acoger la propuesta de implementación del modelo en sus ciudades. Los hechos particulares de la llegada de puesta en marcha de estos sistemas se orienta, como se pudo observar, por un vínculo entre políticas globales y condiciones locales de desarrollo urbano, y esto valida, por otra parte, la 
presencia y el papel preponderante del amplio espectro de actores y organizaciones que intervienen en la gestión y organización de las ciudades contemporáneas.

Lo anterior indica que para estudiar los BRT en América Latina, no basta con la revisión de las condiciones internas o locales que han orientado las políticas de provisión del servicio de transporte público en las capitales a lo largo de las últimas décadas. Por el contrario, en este artículo se muestra que es preciso entender simultáneamente, no solo el problema de la movilidad, sino el conjunto amplio de escenarios del desarrollo urbano en el marco de procesos políticos y económicos de alcance regional y global.

Se observa, en tal medida, que no es casual la presencia en la ciudad de México, Buenos Aires o Bogotá de importantes organizaciones internacionales que promueven el sistema BRT en el mundo, toda vez que de esa manera hacen posible la apertura de espacios de participación que les aseguren visibilidad y, por tanto, oportunidades de expansión de este modelo en los países. Es notable entonces cómo Ciudad de México, por ejemplo, se constituye progresivamente en el centro de operaciones nacional y regional desde donde se comandan las acciones de consultoría y promoción del sistema, y en este proceso el "gran éxito del Metrobús" es la mejor carta de presentación.

\section{REFERENCIAS BIBLIOGRÁFICAS}

AGUILAR VILLANUEVA, Luis F. (2006). Gobernanza y gestión pública. México: Fondo de Cultura Económica.

ALFIE COHEN, Mariam (2009). "Gobernanza: respuesta a las transformaciones del Estado-nación o concepto clave para la reconstrucción de la política". En: Sermeño, Ángel y Serret, Estela (coords.). Tensiones políticas de la modernidad. Retos y perspectivas de la democracia contemporánea. México: UAM-A y Miguel Ángel Porrúa.

ALMANDOZ, Arturo (2008). "Despegues sin madurez. Urbanización, industrialización y desarrollo en la Latinoamérica del siglo XX". En: Revista Eure, agosto, vol. 34, núm. 102.

BALBO, Marcello (2003). "La nueva gestión urbana". En: Jordán, Ricardo y Simioni, Daniela (comps.). Gestión urbana para el desarrollo sostenible en América Latina y el Caribe. Santiago de Chile: CEPAL.

BENKO, George (2000). "Estrategias de comunicación y marketing urbano". En: Revista Eure, diciembre, vol. 25, núm. 79. 
BOISIER, Sergio (2001). "Desarrollo (local): ¿de qué estamos hablando?" En: Madoery, Oscar y Vázquez Barquero, Antonio (eds.). Transformaciones globales, Instituciones y Políticas de desarrollo local. Rosario: Homo Sapiens.

BORJA, Jordi y Castells, Manuel (1999). Local y global. La gestión de las ciudades en la era de la información. México: Taurus.

BULMER-THOMAS, Víctor (2010). Historia económica de América Latina desde la Independencia. México: Fondo de Cultura Económica.

BURGESS, Rod (2003). "Ciudad y sostenibilidad: desarrollo urbano sostenible". En: Cuadernos de la CEPAL, núm. 88.

CALDEIRA, Teresa (2007). Ciudad de muros. Barcelona: Gedisa.

CENTRO DE ESTUDIOS DE FINANZAS PÚBLICAS (CEFP) (2003). Encadenamiento de series históricas del PIB de México. 1970-2001. México: CEFP.

CENTRO DE TRANSPORTE SUSTENTABLE (CTS) (2009). Metrobús: una fórmula ganadora. México: CTS.

(CTS) (2005). “El modelo BRT”. En: Movilidad amble, año 1, núm. 1, septiembre.

COMISIÓN MUNDIAL DEL MEDIO AMBIENTE Y DEL DESARROLLO (1987). Nuestro Futuro Común. México: PNUMA.

CUERVO, Luis M. y González, Josefina (1997). Industria y ciudades en la era de la mundialización. Un enfoque socioespacial. Bogotá: TM Editores.

CUERVO, Luis Mauricio (2005). El falso espejo de la ciudad latinoamericana. Santiago de Chile: Instituto Latinoamericano y del Caribe de Planificación Económica y Social (ILPES) - CEPAL.

DAVIS, Diane (1999). El leviatán urbano. La ciudad de México en el siglo XX. México: Fondo de Cultura Económica.

GUILLAMÓN, David y Hoyos, David (2005). Movilidad sostenible. De la teoría a la práctica. Bilbao: Manu Robles-Arangiz.

DE MATTOS, Carlos (2001). "Transformación de las ciudades latinoamericanas: ¿impactos de la globalización?". En: Revista Eure, diciembre, vol. 28, núm. 85.

DE LA CRUZ, Eugenio (2010). "Reseña del $2^{\circ}$ Congreso internacional de transporte, política y movilidad urbana". En: Transporte y ciudad. Foro de la movilidad, noviembre-diciembre, núm. 02. México: Asociación Méxicana de Transporte y Movilidad (AMTM).

DE OLIVEIRA, Francisco (1977). Elegía para una religión Sudene, Nordeste. Planificación y conflicto de clases. México: Fondo de Cultura Económica. 
DELGADO JIMÉNEZ, Alexandra (2009). "De la participación ciudadana a la gobernanza urbana: transformaciones políticas y territoriales". En: Seminario Un nuevo urbanismo para una sociedad transformada: Alternativas a los nuevos modelos urbanos desde una visión crítica. Sevilla: Universidad Internacional Menéndez Pelayo.

DOMínGUEZ, Olivia (2012). "Los músicos de la informalidad: el Metro como escenario apropiado". En: De Alba, Felipe y Lesamann, Fréderic (coords.). Informalidad urbana e incertidumbre. ¿Cómo estudiar la informalidad en las metrópolis? México: PUEG-UNAM.

DUHAU, Emilio y Giglia, Angela (2004). "Conflictos por el espacio y orden urbano". En: Estudios demográficos y ambientales, mayo-agosto, núm. 56. México: El Colegio de México.

ESCOBAR, Arturo (1998). La invención del tercer mundo. Bogotá: Norma.

GALEANO, Sonia y Beltrán, Arley (2008). Ciudad, informalidad y políticas públicas: Una reflexión desde la sociología de lo cotidiano. En: Cuadernos de vivienda y urbanismo, vol. 1, núm. 2. Bogotá: Pontificia Universidad Javeriana.

GIGLIA, Angela (2010). "Producir y habitar la ciudad informal. Reflexiones desde la antropología". En: Alfie, Miriam et al. (coords.). Sistema mundial y nuevas geografías. México: UAM-A.

GOBIERNO DEL DISTRITO FEDERAL (GDF) (2011). Ciudad de México. Ciudad Global. Acciones locales, compromiso internacional. México: Gobierno del Distrito Federal y Programa Universitario de Estudios sobre la Ciudad-UNAM.

GRAIZBORD, Boris (2011). Sostenibilidad urbana: ¿frase vacía o estrategia de desarrollo urbano? En: Graizbord, Boris y Monteiro, Fernando. Megaciudades y cambio climático: ciudades sostenibles en un mundo cambiante. México: Colmex.

HERNÁNDEZ, Javier (2005). El transporte público concesionado. En: Márquez Ayala, David. El reto del transporte en la ciudad de México. Voces, ideas y propuestas. México: Libros para todos.

ITDP (2010). Guía de planificación de los sistemas BRT. Autobuses de Transito Rápido. ITDP, versión electrónica disponible en http://www.itdp.org/microsites/bus-rapid-transit-planning-guide/guiade-planificacion-de-sistemas-brt-spanish/, página consultada el día 12 de febrero de 2012.

IRACHETA, Alfonso (2005). La necesidad de una política para el desarrollo de Sistemas Integrados de Transporte en grandes ciudades mexicanas. Toluca: El Colegio Mexiquense.

ISLAS, Víctor (2000). Llegando tarde al compromiso. México: El Colegio de México.

JARAMILLO, Samuel (2012). “Urbanización informal: diagnósticos y políticas. Una revisión al debate latinoamericano para pensar líneas de acción actuales". En: Salazar, Clara Eugenia (coord.). Irregular. Suelo y mercado en América Latina. México: Colmex.

LEIDENBERGER, Georg (2011). La historia viaja en tranvía. México: UAM-Cuajimalpa. 
LÓPEZ SALDÍVAR, Ignacio (1999). Nadie está Satisfecho. Los derroteros del transporte público concesionado en el DF. México: Uteha y Acomex.

MONTEZUMA, Ricardo (2010). Movilidad y ciudad del siglo XXI, retos e innovaciones. Bogotá: Universidad del Rosario y Fundación Ciudad Humana.

NAVARRO, Bernardo (2005). "El servicio de transporte urbano y los procesos sociales". En: Revista Veredas, primer semestre.

NORTH, Douglas (1995). Instituciones, cambio institucional y desempeño económico. México: Fondo de Cultura Económica.

OCAMPO, José Antonio (2007). "La macroeconomía de la bonanza económica latinoamericana". En: Revista de la CEPAL, núm. 93.

PARDO, Carlos Felipe (2009). Los cambios en los sistemas integrados de transporte masivo en las principales ciudades de América Latina. Santiago de Chile: CEPAL.

PASSALACQUA, Eduardo (2003). Estrategias e instrumentos de gestión urbana para el desarrollo sostenible en América Latina y el Caribe. Santiago de Chile: CEPAL.

ROGAT, Jorge (2009). Planificación e implementación de un sistema de Bus Rápido en América Latina: resumen orientado a tomadores de decisiones. UNEP, versión electrónica disponible en http://www.unep.org/transport/PDFs/public_transport/BRT_Spanish.pdf, página consultada el día 12 de febrero de 2012.

SALAZAR, Clara Eugenia (2008). "Los corredores confinados de transporte público en las metrópolis latinoamericanas: ¿una oportunidad para hacer ciudad?" En: Salazar, Clara Eugenia y José Luis Lezama (coords.). Construir ciudad. Un análisis multidimensional para los corredores de transporte en la Ciudad de México. México: El Colegio de México.

SASSEN, Saskia (2007). Una sociología de la globalización. Buenos Aires: Kats.

SEIXAS, João (2008). "Dinámicas de gobernanza urbana y estructuras del capital socio-cultural en Lisboa". En: Boletín de la Asociación de Geógrafos de España, núm. 46.

URQUIDI, Víctor (2002). Dimensiones del desarrollo sustentable y el caso de México. En: Cuadernos de trabajo, Programa de Estudios Avanzados en Desarrollo Sustentable y Medio Ambiente (LEADMéxico), núm. 5.

VIVIESCAS, Fernando (1989). Urbanización y ciudad en Colombia. Bogotá: Foro Nacional por Colombia.

VOLVO (2010). "BRT. El bus inteligente". En: $2^{\circ}$ Congreso internacional de transporte, política y movilidad urbana. México: Asociación Mexicana Transporte y Movilidad.

VOUKAS, Yorgos y López, Amilcar (2011). "Ciudad de México". En: Desarrollo urbano y movilidad en América Latina. Panamá: Corporación Andina de Fomento (CAF). 
WINCHESTER, Lucy (2006). "Desafíos para el desarrollo sostenible de las ciudades en América Latina y El Caribe". En: Revista Eure, agosto, vol. 32, núm. 96.

Trabalho enviado em 03 de setembro de 2015.

Aceito em 06 de janeiro de 2017. 